Fig. 2 f. Noch eine Wirbelhöhe tiefer.

Fig. 2 g. Unter dem zweiten Lendennerven.

Fig. 3a. Hund mit Markverletzung; zwisehen dem 3. und 4. Halsnerven. Aufsteigende Degeneration.

Fig. 3b. Derselbe Hund zwischen 5. und 6. Halsnerven. (Degeneration absteigend.)

Fig. 3 c. Zweiter Brustwirbel.

Fig. 3d. Vorletzter Brustwirbel.

Fig. 3 e. Zwischen 1. und 2. Lendennerven.

Fig. 3 f. Aus der Lumbaranschwellung.

\title{
Berichtigender Nachtrag zu meiner Abhandlung über die Erregbarkeit des Rückenmarks.
}

\author{
Zweiter Artikel. \\ Von
}

\section{Se h i f f.}

Die Versuche, auf welche Dittmar (Sächsische Gesellschaft der Wissenschaften 1870 pg. 18) die Ansicht stiitzt, dass gewisse Arten der Reizung des seiner Hinterstränge beraubten Rückenmarks zu Steigerungen des Blutdruckes führen, hatten wir (dieses Arch. Bd. 29 pg. 570) ihrem thatsächlichen Inhalt nach vollständig bestätigen können. Auch einer der wesentlichsten Folgerungen aus diesen Versuchen, dass es sich hier um eine Art Reizbarkeit in Rückenmarksparthien handele, die nach unseren Ergebnissen weder motorisch noch sensibel erregbar sind, glaubten wir unsere Zustimmung nicht versagen zu können, obschon wir (1. e. pg. 578) in Betreff der Zuverlässigkeit unserer Mittel, etwaige Stromesschleifen zu erkennen, uns keineswegs beruhigt zeigten. Rs ist selbstverständlich, dass keines der Mittel versäumt wurde, durch die meine Vorgänger Stromschleifen zu vermeiden oder zu erkennen bemiiht waren, ich bin sogar in dieser Beziehung noch weiter gegangen und habe die so schwer zu lokalisirenden Induktionsströme oft durch Kettenströme ersetzt, die durch Valen- 
tins hernmenden Stromwender diskontinuirlich und alternirend gemacht wurden (dieses Arch. Bd. 29 pg. 578). Von der mechanischen Reizung durch Streichen in der Längsrichtung des Markes, von der mein Vorgänger Erfolge erzielte, wurde in meinen Versuchen fast ganz Abstand genommen, da ich sie nicht in der Weise anwenden durfte, wie ich sie (l. c. pg. 343) als allein zulässig erkannt, wenn Zerrung des noch unverletzten Markes verhindert werden sollte. Die ohne ausgedehntere Zerrung noch mögliche mechanische Reizung ist in ihrem Erfolge zu vorïbergehend, zu momentan, als dass sie den von Dittmar geforderten Bedingungen hätte entsprechen können, die mit Recht während mehrerer Sekunden fortgesetzte Reizungen verlangen.

Der stromanzeigende Froschschenkel bietet bei Versuchen am Riickenmark der Säugethiere wohl iiberhaupt nicht die Vorziige, durch die er sich bei Versuchen an präparirten Fröschen oftmals als unentbehrliches Hülfsmittel erwiesen hat. Als ich zuerst die Versuche Dittmars wiederholte und variirte, hatte ich schon die Unzuverlässigkeit der Froschnerven erkannt, glaubte aber, dass die von mir beobachtete rasch eintretende Unempfindlichkeit besonders den von mir benutzten italienischen Fröschen eigenthümlich sei. In neuerer und neuester Zeit finde ich, dass auch die Genfer Frösche in dieser Beziehung nicht viel voraus haben.

Bedenkt man, dass Versuche iuber sensible Reaktion am Rïckenmark nur in längeren Intervallen wiederholt werden können, und dass der Froschnerv während der Wartezeit in der Tiefe der feuchtwarmen Wunde in einer Temperatur liegen muss, die seine Empfänglichkeit für Reize in hohem Grade beeinträchtigt, dass er ausserdem oft mit dem nie ganz ferne zu haltenden warmen Blute benetzt wird, so begreift man, dass seine Reizbarkeit steil herabsinkt.

Es ist aber nicht allein das Sinken der Reizbarkeit, welches den Gebrauch des Froschschenkels als Anzeigers von Stromschleifen bei Versuchen am Rückenmark der Säugethiere beeinträchtigt. Jeder elektrische Strom wird hier Stromschleifen in grosser Mannichfaltigkeit erzeugen, und es handelt sich nur darum, diejenigen zu erkennen, welche schädlich werden, $d$. h. nicht beabsichtigte Reizungserscheinungen bewirken könnten. In dem dicken Cylinder des Hunderïckenmarks können, der besser leitenden Längsrichtung der Nervenfasern folgend, sich noch Ströme in das Innere 
des Hinterstranges ergiessen, stark genug, um diesen so überaus reizbaren Theil zu erregen, ohne dass sich dieselben gleichzeitig und in gleicher Stärke in den nur äusserlich und gewöhnlich (wegen der Kürze des blosliegenden Theiles) schief aufliegenden, durch Serum, Membranen und etwas Blutflüssigkeit von ihm getrennten Froschnerven ergiessen können. Bei der Verschiedenheit der Widerstände vertheilt sich der derivirte Strom nicht gleichmässig in den Hinterstrang und dem ihm aufliegenden Froschnerven, sondern in dem Hinterstrang ergiesst sich eine Stromschleife und eine sekundäre Schleife dieser letzteren fliesst in dem Froschnerven.

Das erklärt eine auffallende Thatsache, von der sich jeder leicht uiberzeugen kann. Man lege einem tief ätherisirten Hunde das Riickenmark in einer Länge von etwa 3 Centimeter blos, und entferne den Hinterstrang in der hinteren Hälfte der blosgelegten Strecke. Man lasse das Thier aus der Aetherbetäubung theilweise aber nicht vollständig erwachen und bringe einen stromanzeigenden Froschnerven auf den Hinterstrang des vorderen Theiles des blosgelegten Markes. Reizt man jetzt mit allmählich verstärkten Induktionsströmen und sehr genäherten Elektroden die blosgelegte graue Substanz etwa 3 bis 4 Millimeter unter der Einschnittsstelle des Hinterstranges, so wird man in der Regel finden, dass noch ehe die Rollen so weit genähert sind, dass Bewegungen im Froschfusse entstehen, sich schon der Uebergang des Stromes auf die Hinterstränge durch mehr oder weniger energische, aber stets unverkennbare Zeichen kundgibt.

Leider blieben auch andere Versuche erfolglos, in denen ich hoffte den Capillarelektrometer zur Erkennung von Stromschleifen zu verwenden. Allerdings ist dieses Instrument, dessen 'Gebrauch selbst im Vivisektionszimmer sich durch so grosse Bequemlichkeit empfiehlt, oft, aber nicht ohne Ausnahme, viel empfindlicher als der Froschnerv, wo es sich um anhaltende durch das Blitzrad oder einen Invensor unterbrochene Kettenströme handelt. Beim Gebrauch der Induktionsströme ist hingegen der frische Froschnerv bei weitem ein besseres Rheoskop fiir Stromesschleifen ').

Fuir Markstrecken die der Hinterstränge entblösst sind, ist allerdings die Methode, welche ich in der zweiten Abtheilung

1) Herrn Prof. C. Lovén in Stockholm, von dem ich den ersten Kapillarelectrometer erhalten, sei bei dieser Gelegenheit mein verbindlichster Dank ausgesprochen. 
(dieses Arch. Bd. 29 pg. 555) beschrieben, die beste und einfachste ja wie mir scheint die allein zuverlässige, um die Wirkung von Stromschleifen zu erkennen. Diese Methode fordert aber, dass das Mark auf eine lange Strecke hin blosgelegt werde. Dies ist nun mit grösserem Blutverlust verkntipft, und diesen sucht man natürlich zu vermeiden, wo Prüfung einer irritativen Blutdrucksteigerung das Objekt der Untersuchung ist.

Ich glaubte also auch auf dieses Mittel verzichten zu muissen und mich dabei beruhigen zu können, dass, wie direkte Reizversuche am Hinterstrang lehren, bei Versuchen uber reflektorische Blutdrucksteigerung nicht die äusserst ängstliche Vorsorge gegen die geringsten Stromschleifen erforderlich ist, wie sie z. B. bei Versuchen iuber Pupillenerweiterung geboten ist ${ }^{1}$ ).

Denn ein direkt auf den Hinterstrang: wirkender, nicht den Eintritt einer Nervenwurzel berührender und sehr langsam gesteigerter Induktionsreiz bewirkt schon eine Erweiterung der Pupille ehe noch die beiden Rollen so weit genähert sind, dass eine Erhöhung des Blutdruckes entsteht. Bei Anstellung dieses Versuches wurde die Rolle ruckweise jedesmal um 5 Millimeter verschoben und in jeder Stellung mindestens 8 Sekunden lang festgehalten, während ein Gehülfe die Pupille beobachtete. Der Blutdruck wurde mit dem Sphygmoskope aufgeschrieben, das erst dann mit dem Mareyschen Hebel in Verbindung gesetzt wurde, als der mittlere konstante Blutdruck schon ausgeglichen war. Nur die Veränderungen des letzteren brachten also den Hebel aus seiner Horizontalstellung, was natürlich seine qualitative Empfindlichkeit für Druckschwankungen erhöhte. Die Pinselelektroden berührten die Mittellinie der Hinterstränge.

Auf diese Erfahrungen gestiitzt, glaubte ich durch die Wahl schwacher Ströme bei sehr genäherten Polen, durch sehr schwaches Curarisiren der (zuerst natïrlich ätherisirten) Thiere, wodurch eine Reizung im oberen Theil des Markes sich noch durch Zuckungen in den von den dort austretenden vordern Wurzeln versorgten Muskeln kundgeben konnte, und endlich durch supplementäre Anwendung des Froschfusses, mich gentigend vor Stromesschleifen

1) Diesem gegenüber hatte schon Grünhagen in einer Dissertation von Hurwitz darauf hingewiesen, dass nach Reizung die Pupillenerweiterung und die Drucksteigerung wesentlich parallel gehen. 
gesichert zu haben, die noch vom Hinterstrang aus erhöhend auf den Blutdruck wirken könnten.

Und hierin irrte ich, ich hatte die Empfindlichkeit des Hinterstranges für Stromschleifen unterschätzt.

$\mathrm{Zu}$ dieser Erkenntniss gelangte ich zunächst auf folgendem Wege. Ich wollte, wie früher schon Dittmar, im Lanfe des vorigen Herbstes untersuchen, welcher Bestandtheil des seiner Hinterstränge beraubten Rïckenmarks der Sitz der Reizbarkeit sei, vermöge deren der Blutdruck reflektorisch gesteigert wird. Dittmars wenige Versuche, durch die er zu der Ansicht gelangte, dass es der Seitenstrang sei, sind nichts weniger als überzengend. Ich habe deshalb mehreren Hunden den Hinterstrang in einer Strecke von 3 bis $3 \frac{1}{2} \mathrm{~cm}$ resezirt und zuerst den Einfluss der sogen. Kleinhirnseitenstrangbahnen geprüft, deren Verlauf, wie allgemein angenommen wird, vermuthlich ein centripetaler ist. Dieselben je in einiger Länge zu isoliren ist nicht möglich, ich habe sie aber nach der in meinem Aufsatz über die Erregbarkeit angegebenen Methode vorn in der Nähe des obern Wundwinkels durchschnitten und das aus dem Aetherrausch wieder erwachte Thier entweder sogleich oder nach mehreren Stunden sowohl elektrisch im Bereich der Markwunde, als mechanisch an den Hinterextremitäten gereizt, und die Gefässreflexe waren geniigend erhalten.

Bei anderen Hunden mit resezirtem Hinterstrang wurde im Bereich der präparirten Markstelle ein das ganze Mark spaltender Schnitt in der Mittellinie gemacht, und von den beiden Enden desselben zwei Querschnitte dureh die linke Markhälfte geführt. Letztere wurde herausgenommen, die Wurzeln waren beiderseits schon vorher getrennt worden. Nachdem etwas Blut von der medianen Schnittfläche mit einem Schwämmchen aufgesogen worden, gelang es die oberste Spitze des rechten Vorderstrangs von der aufliegenden vorderen Kommissur zu unterscheiden. An der Grenze beider wurde ein kleines Messerchen schief nach vorn und aussen so eingestochen, dass der Vorderstrang von der schmalen Klinge nach innen und unten lag. Das Messerchen fortschiebend wurde so der Vorderstrang isolirt und dann wurde in geeigneter Weise Seitenstrang und graue Substanz am obern und untern Wundende durchschnitten. Das Mikroskop zeigte später, dass dem so isolirten Vorderstrang nach aussen noch ein Minimum grauer Substanz anlag. Reizungen wie oben ausgefuihrt erwiesen 1) dass 
im Vorderstrang keine Fasern verlaufen, deren Reizung centripetal auf den Blutdruck einwirkt, 2) dass auch ron den unterhalb der Wunde gelegenen Theilen vermittelst des Vorderstranges keine Reflexe oder Empfindungen, die den Blutdruck steigern, nach oben geleitet wurden. Nach diesen Versuchen durfte ich dies letztere eigentlich blos für die ersten Stunden nach der Operation behaupten, aber andere Versuche stehen mir zur Seite, in welchen auch bei wochenlangem Ueberleben des Thieres keine Empfindung durch den Vorderstrang oder die Vorderstränge zu Stande gekommen war ${ }^{1}$ ).

In andern Versuchen wurde das Mark auf eine längere Strecke bis zu $6 \mathrm{~cm}$ der Hinterstränge entblösst, dann wurde wie oben die linke Seitenhälfte und der rechte Vorderstrang in der ganzen Länge entfernt. Mittelst einer nach vorn sehr verbreiterten Pinzette wurde jetzt der linke Schnittrand der Dura mater in die Höhe gehoben, so dass die innere mit grauer Substanz bedeckte gewölbte Fläche des Seitenstrangs etwas nach oben sah und nun wurde mit grösster Vorsicht, sanft schabend alle graue Substanz entfernt. Der so in grosser Länge isolirte Seitenstrang wurde wieder in die Wunde versenkt und das erwachende Thier wurde mehrere Stunden sich selbst überlassen. Vor der Prifung mit dem Blutdruckmesser wurde der Hund schwach kurarisirt. Die Prüfung selbst wurde so vorgenommen, dass man zuerst den Seitenstrang ganz nahe dem obern $W$ undwinkel mit allmählich verstärkten Weehselströmen reizte, bis eine leichte Wirkung auf Pupille und Blutdruck zu Stande kam. Die Ströme waren jetzt schon relativ stark, sehr deutlich auf der Zunge fühlbar, die Entfernung beider Rollen 16 bis $19 \mathrm{~cm}$. Dann ging man mit derselben Reizung am Seitenstrang etwas nach hinten, der Wirkung auf den Blutdruck verschwand bald (nach etwa $1 \mathrm{~cm}$ bis $1 \frac{1}{2}$ ) und nachdem man sich dem hintern $W$ undende um $2 \mathrm{~cm}$ genähert hatte, war auch keine Spur von Pupillenwirkung mehr sichtbar. Etwas hinter der Mitte der isolirten Strecke konnte der Seitenstrang mit ganz auffallend starken Strömen misshandelt werden, ohne dass eine Erhöhung des Blutdrucks sichtbar wurde, auch wenn man die Reizung ziemlich lange anhalten liess. Die Wirkung, die also

1) Allerdings waren bei diesen letzteren Versuchen die Vorderstränge an den Kanten aber nur ganz unbedeutend verletzt. (Untersuch. mit Polarisationsapparat.) 
am obern Wundwinkel vom Seitenstrang aus erlangt werden konnte, beruhte in diesen Versuchen evidentermassen auf Stromschleifen.

Es blieb nun noch die graue Substanz. Ich reizte sie nicht isolirt, sondern liess sie in Verbindung mit dem rechten Vorderstrang, dessen Unwirksamkeit ja bereits erkannt war, und auch vom Seitenstrang liess ich die buchtig zwischen Vorder- und Hinterhorn sich einschiebende Parthie. Nach derselben Methode wie für den Seitenstrang wurde erkannt, dass ihre selbst sehr kräftige Reizung nicht erhöhend auf den Blutdruck einzuwirken vermag, wenn wir uns gegen die täuschende Wirkung der Stromesschleifen nach der 1. c. p. 555 angegebenen Methode zu wahren verstehen.

Man begreift, dass ich mich nicht leicht bei diesen Resultaten beruhigte, dass ich die Versuche oft und öfter wiederholte, aber endlich sprachen sie klar und überzeugend genug.

Also alle einzelnen Bestandtheile des der Hinterstränge entblössten Dorsal- oder obern Lendenmarks, die Vorderstrünge, die graue Substanz und die Seitenstränge waren, von Stromschleifen abgesehen, noch einige Stunden nach der Operation der direkten Reizung unzugänglich, wirkten nicht auf den Blutdruck. Wie soll man es erklären, dass die genannten Theile zu e in em Strange vereinigt eine Wirkung zeigten, die jedem einzelnen derselben fehlt.

Ehe es gestattet war eine schwankende hypothetische Basis zur Erklärung herbeizuziehen, schien es mir geboten nochmals zu untersuchen, ob nicht die Wirkung der vereinten Stränge dennoch auf Stromschleifen zurückzuführen sei. Wie hier die Seitenstrünge und die graue Substanz war das ganze blosgelegte Rückenmark friiher nicht untersucht worden, weil man gefurchtet hatte, durch zu ausgiebige Bloslegung den Blutdruck zu sehwächen. Jetzt hatte es sich bei den Versuchen an den Seitensträngen gezeigt, dass diese Besorgniss übertrieben war. Ich hatte trotz der ausgedehnten Bloslegung doch noch den Blutdruck über 100 oder 110 bewahren können.

Allerdings ist es für die isolirten Stränge leichter Stromesschleifen zu überwinden und zu erkennen, als für das dickere Mark, weil mit der abnehmenden Dicke der Widerstand für die Schleifen mehr wächst als der Widerstand der direkten Verbindungslinie zwischen den Elektroden. Aber die Methode der successiven Verlängerung der Schleifenbahn bei gleichbleibendem direktem Reiz wird auch für die vereinigten Stränge wenn auch 
langsamer, dasselbe leisten mitssen, wie für den Seitenstrang. Hatte sich doch schon früher unter ähnlichen Verhältnissen diese Methode für das Studium der Pupillenerweiterung bei Markreizung trefflich bewährt. Allerding's sind hier die letzten verschwindenden Spuren einer Wirkung dentlicher und rascher zu erkennen, als bei Blutdruckschwankungen. Die Ströme durften daher so schwach gewählt werden, dass schon wenige Millimeter vom Hinterstrang entfernt ihre Wirkung undeutlich zu werden begann, um bei weiterem Herabrïcken ganz zu schwinden.

Ich ging also nochmals an den Versuch. Jungen Hunden von nicht zu kleiner Statur wurde in tiefster Aethernarkose das Riuckenmark auf die Länge von etwa $6 \mathrm{~cm}$ blosgelegt. Die Hinterstränge wurden entfernt und dann wurden die Thiere in zwei Gruppen vertheilt. Einigen derselben wurde schon während des Erwachens, sobald die Blutung gestillt war, Curare in schwacher Dosis gegeben und die Carotis wurde, sobald die künstliche Athmung eingeleitet war, mit dem Manometer verbunden. Die andern Hunde wurden nach Vernähen der Wunde mehrere Stunden aufbewahrt, bis die Empfindung, resp. die Hyperästhesie in den Hinterfüssen sich deutlich kundgab, zum Beweise, dass die entblösste Markstelle bei der Operation nicht gelitten hatte. Erst dann wurden sie curarisirt und wie die vorigen behandelt. Ausser den Metallelektroden, die blos bei den letzten Versuchen dienten, wurden zur Schonung des Markes auch Pinselelektroden (Fleischl) in genau gleichbleibender wechselseitiger Entfernung angewendet. Der ausschliessliche Gebrauch der letzteren hätte zu dem Einwurfe Veranlassung gegeben, dass in den aufeinander folgenden Versuchen die Entfernung der in der Tiefe der Wunde sich umbiegenden Spitzen nicht dieselbe geblieben sein dürfte. Im obersten Wundwinkel, ganz in der Nähe des Hinterstrangquersehnittes wurde die Stromstärke absichtlich etwas zu hoch gewählt, so dass deutliche und ziemlich rasche (2 bis 4 Sekunden) Erhöhung des Druckes und plötzliche Erweiterung der Pupille entstand ${ }^{1}$ ). Man rïckte nun mit den Elektroden herab und man war noch merklich weit von der Mitte der Wunde entfernt, als die Wirkung anf Blutdruck und auf die Pupille gänzlich aufhörten. Ja man durfte sogar die Rollen etwas näher aneinanderrücken, ohne dass sie wieder erschienen.

1) Auch die Schnelligkeit der Pupillenerweiterung wächst bei undurchschnittenem Halsvagus mit der Heftigkeit der sensiblen Reizung. 
Bei dieser Gelegenheit bemerkte ich, dass man sich mit dem Reize dem hinteren Wundwinkel weit mehr als dem vorderen nähern durfte, obne dass Zeichen der Reizbarkeit auftraten, diese Eigenthümlichkeit fand sich bei den meisten, nicht bei allen Hunden, die zu diesen Versuchen dienten. Bei zwei Hunden war sie nicht wahrzunehmen.

Nachdem die Versuche in dieser Weise vom obern Wundwinkel nach unten fortschreitend vorgenommen waren, wiederholte man sie mit entsprechendem Erfolge in umgekehrter Richtung. Dies zum Beweise, dass die Erfolglosigkeit der Reizung in der mittleren Strecke der Wunde nicht etwa daher riihrte, dass die vorhergehende Reizung der oberen Parthie die Leitung in derselben beeinträichtigt hätte.

Die entblössten Rückenmarksstrecken entsprechen nicht in allen Versuchen genau denselben Wirbeln. Das obere Ende der Wunde traf bald anf den vorletzten, den letzten Rückenwirbel oder den ersten Lendenwirbel. Dies zum Beweise, dass der wirkungslose Reiz nicht immer eine bestimmte Strecke des Markes getroffen, die, wie man argwöhnen könnte, sehon an und für sich weniger erregbar wäre.

Diesen Thatsachen gegenüber konnte ich mich nicht mebr weigern zuzugestehen, dass ich mich in meinen Versuchen von 1870 durch Stromschleifen habe täuschen lassen, und dass in der That, wie ich dies vorher immer behauptet, die Hinterstränge die einzigen reizbaren Theile des Markes seien.

Doch wollte ich auch noch eine andere Methode versuchen, die der polaren Reizung Brenners analog von Chauveau (Comptes rend. Vol. 81 pg. 779) zuerst unter dem Namen der un ipolaren Erregung 1875 in die physiologische Methodik eingeführt, dann in etwas verschiedener Form von Gergens und von Tiegel (dieses Areh. Bd. 12 u. 13) benutzt und endlich in neuerer Zeit von Kühne zum Zwecke einer sehr genauen Lokalisirung der Reizung warm empfohlen wurde (Heidelberger Untersuchungen IIT. pg. 21). Kühn e führt bei dieser Gelegenheit an, dass er diese Reizmethode bereits 1860 erprobt habe (Müllers Arch. 1860 p. 483) ${ }^{1}$ ). Ich verfuhr in folgender Weise. Der eine Pol (der weniger reizende) eines Schlitteninduktors mit Eisendrahtkern und ohne

1) Dieses Citat entnahm in den Heidelberger Untersuchungen ohne es verglichen zu haben. 
Helmholtz'sche Vorrichtung wird mit der Gasleitung verbunden, der andere in eine gläserne Handhabe gefasst. Das Thier kommt auf eine sehr grosse (etwa 2 Meter lange, $80 \mathrm{~cm}$ breite) Zinkplatte, die auf Holzftissen rubt. Auf die Zinkplatte ist eine wässerige Lösung gegossen, mit der auch die Bauchseite des Thieres eingerieben wird, um eine leitende Verbindung herzustellen. Wird nun irgend ein entblösster Theil des Thieres, während der Hammer des Induktoriums spielt, mit dem in Glas gefassten Poldrathe berihrt, so ergiesst sich von der Contaktstelle ein Strom nach allen Richtungen. Denselben kann man so weit schwächen, dass er in beliebiger Entfernung von der Elektrode nicht mehr dicht genug ist, um auch nur die geringste Reizung zu bewirken, während er am Contaktpunkte selbst noch ziemlich stark und oft relativ sehr mächtig ist. Man kann nun annähernd bestimmen, wie weit im einzelnen Falle die reizende Wirkung um die Elektrode herum sich erstrecken darf. Vor dęr Markreizung wählte ich mir einen etwa in der Richtung seiner Fasern durchschnittenen Riickenmuskel. Die Elektrode wird ihm aufgesetzt und die sekundäre Spirale des Induktoriums wird dann so weit verschoben, dass auf dem Muskel eine Kontraktionsfurche von etwa 4 Millimeter Breite entsteht. Die Deviation im Muskel ist also nach jeder Seite nur 2 Millimeter weit wirksam, und im Rückenmark wird ihre Wirksamkeit nicht sehr bedeutend weiter gehen. Es wird nun der Pol der entblössten Stelle des Markes .etwa $6 \mathrm{~mm}$ von dem Durchschnitt der Hinterstrünge entfernt angelegt. Man bertihrt die Seitenstränge, die Hinterhörner, die centrale graue Substanz und es entsteht keine Spur von Wirkang. Die Pupille erweitert sich nicht, der Blutdruck hebt sich nicht.

Geht man aber $2 \mathrm{~mm}$ weiter nach oben so entsteht eine ganz schwache Erweiterung der Pupille, rlickt man noch einen Millimeter herauf, so ist die Erweiterung stärker und die Wirkung auf den Blutdruck beginnt. Die ganze weiter unten gelegene entblösste Strecke des Markes bis ganz nabe dem hinteren Wundwinkel zeigt natürlich keine Wirkung. Und dabei ist die Wirkung des Poles an der Zangenspitze noch merklich stark.

Macht man nach Aussage der Muskelprobe die Reizung stärker, so dass die tetanische Furche etwa 6 bis $7 \mathrm{~mm}$ breit wird, so muss man sich in der Markwunde etwas weiter von den Hintersträngen entfernen, um keine Wirkung zu haben. So ist es mir 
nach vorläufiger Beobachtung am Muskel mebrfach gelungen, den beobachtenden Assistenten vorherzusagen, ob die nächste von mir vorzunehmende Reizung eine stärkere, eine schwache oder gar keine Wirkung haben werde. Natuirlich hat man bei so eng und nach Belieben enger lokalisirten Reizung keine langen Markstrecken bloszulegen, was dem Blutdruck und der Promptheit seiner Antworten zu Gute kommt.

Da die Probe an meiner Zungenspitze für die Stärke der punktförmigen Reizung an dem nahezu isolirten Thiere eigentlich ohne wahre Bedeutung ist, habe ich in einigen Versuchen das aus dem Aetherrausch wieder erwachte Thier nur sehr schwach curarisirt. Von den der präparirten Markstelle entsprechenden Nervenwurzeln hatte ich blos die hinteren durchschnitten. Der Reiz wurde so weit verstärkt, dass er anf dem Muskel etwa $5 \mathrm{~mm}$. wirksamer Deviation gab. Als nun etwa die Mitte der präparirten Markstelle durch ganz oberflächliches Einstechen der Nadelelektrode gereizt wurde, kontrahirten sich schnell und kräftig die dem hier abgehenden Nerven derselben Seite entsprechenden Rumpfmuskeln, aber jede Wirkung anf den Blutdruck blieb aus. Ging man höher oder tiefer gegen die durchschnittenen Hinterstränge, so entstand eine erst leichte und langsame, mit der Annäherung an den Wundwinkel wachsende Erhöhung des arteriellen Druckes. War man den Hintersträngen sehr nahe (etwa 2 bis $1 \frac{1}{2} \mathrm{~mm}$ ) gekommen, so entstanden auch rudimentäre Reflexbewegungen in entfernten Rumpfmuskeln und besonders in den Hautmuskeln. Man sieht also, dass die Reizung eine relativ sehr kräftige war, demn ein Reiz, der die Wurzeln der motorischen Nerven kräftig erregen kann, ist für die Hinterstränge schon ein übermächtiger.

Wir kehren also zu der von mir schon im Lehrbuch der Nervenphysiologie ausgesprochenen und lange festgehaltenen Ansicht zurück, dass alle Fasern der Vorderstränge, Seitenstränge und der grauen Substanz, abgesehen von den intramedullaren Wurzeln der motorischen Nerven, wur ästhesodische und kinesodische, äusseren Reizen nicht zugängliche sind.

Der Unterschied aber den ich (dieses Arch. Bd. 29 pg. 577 bis 579) zwischen der von dem der Hinterstränge entblössten Marke (durch Stromschleifen) und der von den Hintersträngen direkt erzeugten Druckerhöhung angegeben, hat sich mir auch bei meinen neueren Versuchen immer bewährt, wenn ich nicht absichtlich all-

E. Pfüger, Archiv f. Physiologie. Bd. XxxI. 
zu starke Stromschleifen einwirken liess. Eine ganz schwache Reizung der Hinterstränge - und eine solche ist die durch unabsichtlich entstehende Stromesschleifen bewirkte - erzengt in der That den wahrnehmbaren Gefässreflex nur vermittelst des verlängerten Markes, eine direkte oder etwas stärkere wirkt auf den Blutdruck auch durch spinalen Reflex, wenn das obere oder auch das mittlere Halsmark durchsehnitten sind.

\section{Ueber die Verschiedenheiten des Eiereiweisses bei befiedert geborenen (Nestflüchter) und bei nackt geborenen (Nesthocker) Vögeln}

und

\section{Ueber die Verhältnisse zwischen dem Dotter und dem Eiereiweiss.}

Von

\section{Prof. J. Tarchanoff}

in St. Petersburg.

In der jetzigen Mittheilung will ich nur in Kürze die Resultate meiner im verflossenen Sommer durchgeführten Untersuchungen uber das Eiweiss und den Dotter von verschiedenen Vögeln mittheilen.

I. In frischen Eiern von nackt und blindgeborenen Vögeln (Uferschwalbe, Hänfling, Fink, Drossel, Kanarienvogel, Taube, Krähe, Saatkrähe, Nachtigall, Rothschwänzchen, Sperling) befindet sich eine besondere Art von Eiereiweiss: dasselbe bleibt, nachdem es durch das Kochen coagulirt ist, vollkommen durchsichtig und gallertartig und folglich zeigt es stark ausgesprochene Verschiedenheiten im Vergleiche mit dem gewöhnlichen weissen gekochten Eiereiweiss des Huhnes. Das Eiweiss der letzten Art findet sich bei allen denjenigen Vögeln vor, welche befiederte Junge aus den Ejern ausbrüten (Huhn, Kalkun, Ente, Gans, Perlhubn, Feldhuhn, Wiesenschnarre u. s. w.). Bis jetzt habe ich nur eine Ausnahme von dieser Regel gefunden, nämlich den Kibitz, 